\title{
Parámetros urbanos morfo-materiales y su correlación con las temperaturas de aire en verano
}

\author{
Urban morpho-material parameters in cities and their \\ correlation with summer air temperatures
}

\begin{abstract}
Noelia Liliana Alchapar
Cláudia Cotrim Pezzuto

Erica Norma Correa

\section{Resumen}

$\mathbf{C}$

on el objetivo de determinar y comparar la eficiencia de diversas estrategias de enfriamiento urbano en dos ciudades Latinoamericanas (Mendoza, Argentina y Campinas, Brasil) fueron analizados 18 escenarios que modifican su porcentaje de vegetación y nivel de albedo en materiales opacos de la envolvente urbana. Los escenarios fueron simulados con un modelo de alta resolución microclimática - ENVI-met 3.1. Mediante el Análisis de Componentes Principales se evaluaron las posibles asociaciones entre variables. Como resultado se observó que el porcentaje de vegetación y el albedo medio de las superficies opacas urbanas explica el 71,5\% de los comportamientos dentro de los canales viales evaluados en ambas ciudades. El incremento de vegetación podría disminuir la temperatura media en Mendoza hasta $2,0^{\circ} \mathrm{C}$, mientras que en Campinas hasta $5,0^{\circ} \mathrm{C}$. Aumentar el nivel de albedo en la envolvente urbana eleva hasta $1,0^{\circ} \mathrm{C}$ las temperaturas de aire en escenarios de alta densidad de Mendoza y Campinas. No obstante, en el caso de los escenarios con alto albedo en superficies horizontales y bajo albedo en verticales, se registraron reducciones de $2,0^{\circ} \mathrm{C}$ en la ciudad de Mendoza y de $3,0^{\circ} \mathrm{C}$ en Campinas.

Palablas-clave: Temperaturas urbanas. Análisis multivariado. Vegetación urbana. Albedo de materiales.

${ }^{1}$ Noelia Liliana Alchapar Instituto de Ambiente, Hábitat y Energía Mendoza - Argentina

${ }^{2}$ Cláudia Cotrim Pezzuto 2Pontifícia Universidade Católica de Campinas Campinas - SP - Brasil

${ }^{3}$ Erica Norma Correa ${ }^{3}$ Instituto de Ambiente, Hábitat y

Energía

Mendoza - Argentina

Recebido em 21/08/17 Aceito em 23/11/17

\section{Abstract}

In order to determine and compare the efficiency of different urban cooling strategies in two Latin American cities (Mendoza, Argentina and Campinas, Brazil), 18 scenarios that modified their percentage of vegetation and albedo level in urban envelopment materials were analysed. The scenarios were simulated using a high-resolution microclimatic model-ENVI-met 3.1. The possible associations between variables were evaluated through Principal Components Analysis. The result showed that the percentage of vegetation and the average albedo of the urban opaque surfaces explain $71.5 \%$ of the behaviours within the urban canyons in both cities. The increase in vegetation could decrease the average temperature in Mendoza by up to $2.0^{\circ} \mathrm{C}$, while in Campinas by up to 5.0 ${ }^{\circ} \mathrm{C}$. Increasing the albedo level in the urban envelopment raises the air temperatures in high-density scenarios of Mendoza and Campinas by up to $1.0^{\circ} \mathrm{C}$. However, in the case of high albedo scenarios in horizontal surfaces and low albedo in verticals, there were reductions of $2.0^{\circ} \mathrm{C}$ in the city of Mendoza and 3.0 ${ }^{\circ} \mathrm{C}$ in Campinas.

Keywords: Urban temperatures. Multivariate analysis. Urban vegetation. Albedo materials.
\end{abstract}




\section{Introducción}

El hecho de que las ciudades son más cálidas que sus entornos rurales ha sido investigado por décadas (UNITED..., 2015). En las ciudades de clima templado el sobrecalentamiento exacerba la demanda adicional de energía (AC). Este aumento de temperatura agrava el consumo de energía para refrigeración y la demanda eléctrica en horas pico. Así como también intensifica los problemas de polución y la huella urbana, causando disconfort ambiental y problemas en la salud humana (MIRZAEI; HAGHIGHAT; 2010, KOLOKOTRONI; GIRIDHARAN, 2008).

La combinación del aumento del consumo energético y la modificación en el balance radiativo provocan un incremento de la temperatura urbana entre $1,3{ }^{\circ} \mathrm{C}$ y $15,0{ }^{\circ} \mathrm{C}$ (OKE, 1982; ARNFIELD, 1982; GIVONI, 1994). Este incremento depende de varios factores que pueden ser clasificados en incontrolables y controlables (RIZWAN; DENNIS; LIU, 2008). Las variables incontrolables están relacionadas con las características ambientales y topográficas específicas del área (radiación solar, porcentaje de nubosidad, condiciones anticiclónicas y estacionales, velocidad y dirección de vientos, etc). Dentro de las variables controlables, se pueden citar principalmente la liberación de calor antropogénico; almacenamiento excesivo de radiación solar de la materialidad edilicia, disminución de superficies verdes en relación a las superficies selladas, morfología edilicia y vial que obstruye la circulación de aire, entre otras.

Santamouris et al. (2017), evaluaron el potencial de enfriamiento de diferentes tecnologías de mitigación de 220 áreas urbanas. Sus resultados demostraron que los techos y pavimentos reflectivos y el incremento de la vegetación urbana pueden disminuir las temperaturas de aire en una ciudad y contrarrestar los efectos de las islas de calor en parte o en su totalidad. La caída de temperatura registrada en todas las ciudades es de 2,0 ${ }^{\circ} \mathrm{C}$ promedio.

En este sentido, las propiedades ópticas de los materiales (albedo y emisividad) determinan el consumo energético y las condiciones de confort en el interior de los edificios, así como también el confort en los espacios exteriores (SANTAMOURIS; SYNNEFA; KARLESSI, 2011).

Akbari, Matthews y Seto (2012) estimaron el efecto a largo plazo del incremento de albedo en superficies urbanas. En un metro cuadrado de superficie, cada incremento de 0,01 de albedo produce un efecto de enfriamiento de $3 \times 10^{-15} \mathrm{~K}$ y una reducción de emisiones de $\begin{array}{lllll}7 & \mathrm{~kg} & \mathrm{CO}_{2}\end{array}$ equivalente.

Akbari, Menon y Rosenfeld (2009) relatan que el aumento del albedo urbano puede reducir las temperaturas de aire, sin embargo, esta estrategia debe emplearse siguiendo distintos criterios de acuerdo a cada caso en particular.

Otro aspecto que presenta importancia en el control climático urbano es la vegetación (ALITOUDERT, 2005; ABREU-HARBICH; LABAKI; MATZARAKIS, 2014; PEZZUTO, 2007; MUNIZ; PEZZUTO, 2016). La vegetación urbana tiene un gran potencial de refrigeración pasiva y de medida de mitigación de la isla de calor (SHASHUA-BAR; TSIROS; HOFFMAN, 2010, 2012). Estudios relatan el efecto de la atenuación climática causado por parques urbanos y bosques. A meso-escala, Dacanal (2011) afirma que el porcentaje de área de bosque, sobre el total urbanizado, debe ser superior al $20,0 \%$ para que pueda ser verificado el efecto de enfriamiento y de elevación de la humedad relativa del aire. Al evaluar la escala micro-climática, investigaciones desarrolladas por Alchapar, Pezzuto y Correa (2017) demostraron que el incremento de vegetación urbana de 20,0 a $60,0 \%$ puede disminuir la temperatura de aire entre 2,0 y $4,0{ }^{\circ} \mathrm{C}$ en cañones urbanos de la ciudad de Campinas (Brasil) y de Mendoza (Argentina).

Por otro lado, la formación de los ambientes térmicos urbanos también está directamente asociada a los aspectos de la morfología de su entorno, así como por el conjunto complejo de la estructura urbana (PEZZUTO, 2007; MINELLA; HONJO; KRÜGER, 2012; MINELLA; KRÜGER, 2017). Stewart y Oke (2012) afirman que las diferencias de temperatura en el medio urbano son influenciadas por diversos parámetros de la geometría urbana. Investigaciones desarrolladas por Chen et al. (2011) abordan la fuerte relación entre los patrones de uso del suelo y el consumo de energía. Los autores verificaron que tanto la forma urbana como la fragmentación y la irregularidad de los patrones de uso del suelo están positivamente relacionadas con el consumo de energía.

La preocupación por los problemas ambientales ha favorecido al desarrollo de estrategias que propician la reducción de las temperaturas urbanas, sin embargo, pocos estudios abordan el impacto de cada una de ellas sobre diferentes ciudades. Es por ello que en este trabajo se propone detectar, en términos cuantitativos, qué estrategias de enfriamiento urbano son más eficientes en dos ciudades latinoamericanas, Campinas-Brasil y 
Mendoza-Argentina. Estas ciudades tienen comparables patrones de crecimiento y capacidades tecnológicas y económicas.

Con el objetivo dar continuar y profundizar la investigación desarrollada por Alchapar et al. (2017), que evaluaron el comportamiento microclimático de cañones urbanos en la ciudad de Mendoza (Argentina) y Campinas (Brasil), se realiza un análisis de componentes principales (ACP) de datos obtenidos de simulaciones computacionales con el programa ENVI-Met 3.1. El propósito del trabajo es identificar y analizar las posibles asociaciones que existen en canales viales de alta densidad entre la temperatura del aire y parámetros morfológicos (porcentajes de vegetación urbana y factor de visión de cielo) y parámetros tecnológicos (modificación de niveles de albedo de materiales de la envolvente urbana).

\section{Metodología}

Esta sección se estructura de la siguiente forma: selección de las áreas de estudio de cada ciudad y monitoreo de las condiciones microclimáticas en el período de verano, digitalización y ajuste del modelo tridimensional en el programa ENVI-Met 3.1 (ENVI-MET, 2017); simulación de los escenarios de alta densidad propuestos; análisis multivariado por componentes principales.

\section{Selección de áreas de estudio y monitoreo}

Para detectar cuantitativamente cuáles estrategias (porcentaje de vegetación, factor de visión de cielo (FVC) y albedo) resultan más efectivas para disminuir las temperaturas urbanas en distintos contextos climáticos (Mendoza, Argentina y Campinas, Brasil) fueron seleccionados dos recortes urbanos que poseen patrones de morfología urbana comparables (ALCHAPAR et al., 2017).

El área de estudio de la ciudad de Mendoza (S 32 54 '48" S, 68 50'46" O, altitud media 750m) está localizado en el sureste del área metropolitana, con una ocupación horizontal de baja densidad consolidada de uso mixto (residencial e comercial). La relación entre la altura edilicia el ancho del canal vial tiene un aspecto de ratio $\mathrm{H} / \mathrm{W}^{1}$ de 0,30. El área tiene aproximadamente $60 \%$ de

\footnotetext{
${ }^{1}$ La relación de aspecto de ratio $\mathrm{H} / \mathrm{W}$ es un indicador que permite caracterizar la profundidad de un cañón vial y por lo tanto determinar su densidad. Representa la relación dimensional entre la altura edilicia $(\mathrm{H})$ y el ancho del canal vial (W). Cuando el resultado es mayor a 1 significa que la altura edilicia supera el ancho de calle.
}

vegetación en cañones urbanos. Las especies predominantes son de segunda magnitud, entre las que se distinguen morus alba y un menor número de jacarandá mimosifolia y platanus hispánica. La materialidad de la envolvente edilicia en el plano horizontal se compone de hormigón en los pavimentos vehiculares y en los pavimentos peatonales de baldosas calcáreas amarillas, rojas y negras. El $80 \%$ de los techos son planos de losa maciza y membrana y el $20 \%$ restante es inclinado de chapa metálica o tejas cerámicas. En el plano vertical las fachadas son de ladrillo visto, piedra laja oscura, o revoque fino pintados en tonalidades variadas.

El área de estudio de la ciudad de Campinas (S 22 53'20" S, O 4704'40" O, altitud media 680m) está localizado en un área central de la ciudad, en una región de ocupación horizontal de baja densidad consolidada de uso mixto (residencial y comercial). La relación entre la altura edilicia y el ancho del canal vial tiene un aspecto de ratio $\mathrm{H} / \mathrm{W}$ de 0,22 . El área presenta 20,0\% de vegetación en cañones urbanos. Las especies predominantes son de mediana magnitud, entre las que se distinguen sibipiruna "Caesalpiniapeltophoroidesbenth", pata de vaca "Bauhiniavariegata" y sauce de pequeño porte "Salixbabylonica". El trazado urbano es ortogonal, la materialidad de la envolvente en el plano horizontal se compone de asfalto en los pavimentos vehiculares y en los peatonales de piedra calcárea amarilla y rojiza $(50,0 \%)$, y de concreto $(50,0 \%)$. Los techos son inclinados, con predominancia de tejas rojizas y un pequeño porcentaje de chapas metálicas y losas. Los materiales que constituyen las superficies verticales son piedra natural y pinturas en tonos variados.

La evaluación climática en ambas áreas, Mendoza y Campinas se realizó en la estación de verano por ser el periodo más demandante. En la ciudad de Mendoza los registros fueron el 14 de enero (temperatura del aire máxima diaria $36,0{ }^{\circ} \mathrm{C}$, temperatura del aire mínima diaria $21,0{ }^{\circ} \mathrm{C}$ y temperatura del aire promedio $27,0{ }^{\circ} \mathrm{C}$, radiación global media del mes de febrero 303,08 W/m², humedad relativa del aire $42 \%$ ). El monitoreo ocurrió en un día estable, sin precipitaciones. En la ciudad de Campinas la recolección de datos ocurrió el 23 de febrero (temperatura del aire máxima diaria $33,0{ }^{\circ} \mathrm{C}$, temperatura del aire mínima diaria $20,5^{\circ} \mathrm{C}$ y temperatura del aire promedio $26,0^{\circ} \mathrm{C}$, radiación global media del mes de febrero $355,00 \mathrm{~W} / \mathrm{m}^{2}$, humedad relativa del aire $67,0 \%)$

Cabe destacar que los recortes seleccionados representan regiones de baja densidad y proximidad con el centro urbano. 


\section{Simulación y calibración con el programa ENVI-Met}

El microclima de las áreas de estudio fue calculado con el programa ENVI-Met V3.1 que predice las interacciones entre las superficies urbanas, vegetación y atmósfera, a partir de modelos de simulación de los flujos de aire, turbulencias, perfiles de temperatura de aire y humedad relativa al aire y los flujos de radiación (BRUSE; FLEER, 1998). Como las áreas de estudio contemplaron un recorte urbano de $210 \times 210 \mathrm{~m}$, las simulaciones se realizaron con una grilla de 70x70x30 (X, Y, Z), con una resolución de cuadrícula 3 x 3 x 3m.

La Tabla 1 detalla los de configuración del software ENVI-Met. Para la calibración de los datos simulados y medidos fueron recolectadas las variables de temperatura de aire y humedad relativa y radiación solar. Los registros se realizaron mediante estaciones fijas (Punto 2) ubicadas sobre la vereda norte, en postes de luminarias, a 2,5 metros aprox. de altura (Figura 1). Los equipos de medición fueron: $\mathrm{HOBO}^{\circledR} \mathrm{H} 08$ 003-02 (precisión de temperatura: $\pm 0,7{ }^{\circ} \mathrm{C}$ de $+21,0{ }^{\circ} \mathrm{C}$ y precisión de humedad: $\pm 5,0 \%$ de $+5,0$ ${ }^{\circ} \mathrm{C}$ a $+50{ }^{\circ} \mathrm{C}$ ) en Mendoza; y TESTO $174 \mathrm{H}$ (precisión de temperatura: $\pm 0,5{ }^{\circ} \mathrm{C}$ de -20 a +70 ${ }^{\circ} \mathrm{C}$ y precisión de humedad: $\pm 3,0 \%$ de $2,0 \% \mathrm{HR}$ a $98,0 \% \mathrm{RH}, \pm 1$ dígito $+0,03 \% \mathrm{RH} / \mathrm{K})$ en Campinas. Ambos equipos se instalaron dentro de protectores que evitan la incidencia directa de radiación solar y contra la intemperie (HOBO RS1 Solar Radiation Shield).

Tabla 1 - Parámetros de ingreso en el programa ENVI-Met. Mendoza (Argentina) y Ciudad de Campinas (Brasil)

\begin{tabular}{l|c|c|l|c|c}
\hline \multicolumn{1}{c|}{ Datos Principales } & Mendoza & Campinas & \multicolumn{1}{|c|}{ Dados Principales } & Mendoza & Campinas \\
\hline $\begin{array}{l}\text { Velocidad de viento } 10 \\
\text { m (m/s) }\end{array}$ & 3,0 & 1,9 & $\begin{array}{l}\text { Temperatura Inicial } \\
\text { Atmosférica (K) }\end{array}$ & 300,0 & 295,4 \\
\hline $\begin{array}{l}\text { Dirección de Viento } \\
(0: N, 90: E, 180: S, 270: W)\end{array}$ & 150 & 135 & $\begin{array}{l}\text { Humidad Específica 2500 } \\
\text { m (gr agua/kg air) }\end{array}$ & 2,8 & 8,2 \\
\hline Rugosidad & 0,1 & 0,1 & Humidad Relativa 2m (\%) & 28,0 & 65,7 \\
\hline
\end{tabular}

Figura 1 - Área de estudio -Mendoza (Argentina) y Campinas (Brasil)-; Punto 1 (punto de calibración); Punto 2 (punto de análisis)

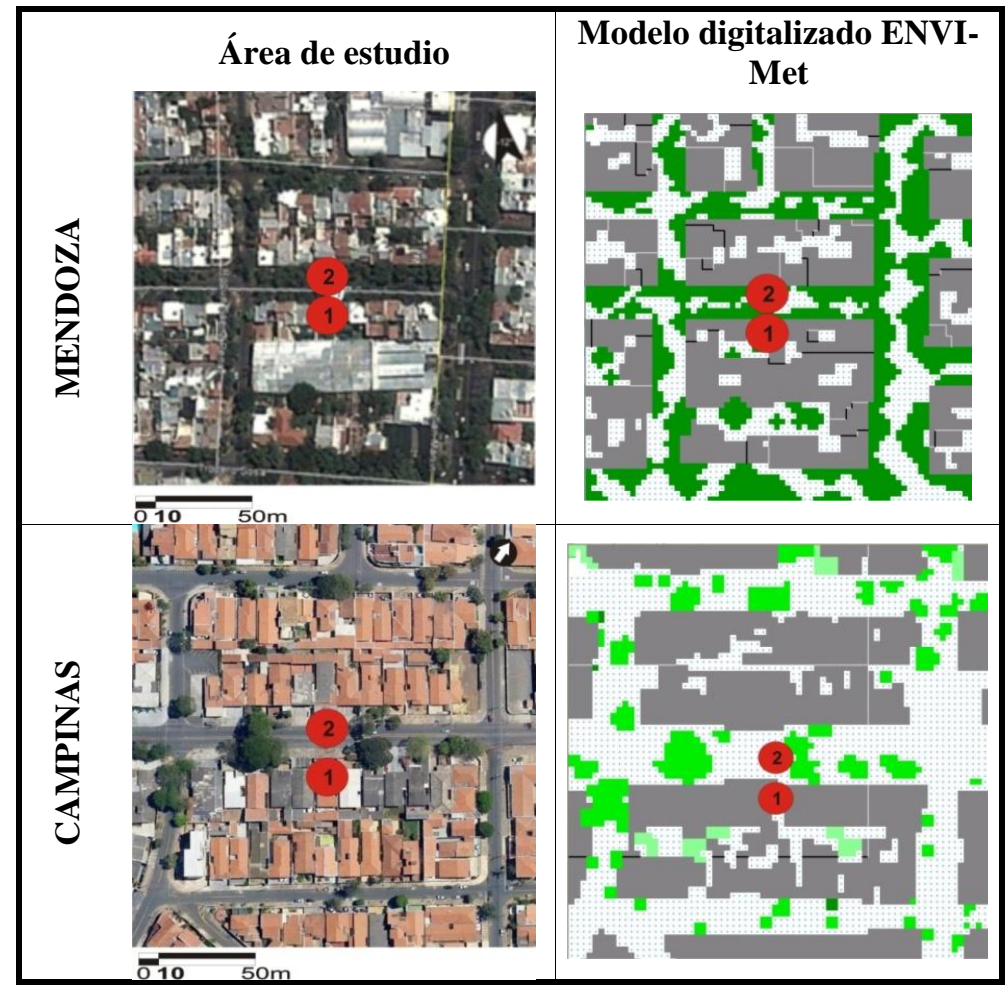

202 Alchapar, N. L.; Pezzuto, C. C.; Correa, E. N. 
Los datos de velocidad y dirección del viento a 10 metros de altura se recogieron a partir de estaciones meteorológicas urbanas de referencias. En la ciudad de Mendoza los datos fueron recolectados desde el Observatorio del Parque General San Martín, aproximadamente a $4 \mathrm{~km}$ noroeste distante del área de estudio. Los datos de la ciudad de Campinas fueron recogidos del Instituto Agronómico de Campinas (IAC), aproximadamente a $2,5 \mathrm{~km}$ noroeste distante del área de estudio. Los datos de humedad específica fueron obtenidos desde el Aeropuerto de Mendoza (Estación número 87418), y del Aeropuerto Campo de Marte (Estación número 83779) para la ciudad de Campinas.

Para el diseño del modelo teórico, la vegetación de cada área fue seleccionada según el índice de área foliar (LAD) disponible en la librería del software ENVI-Met 3.1, dado que las características fisiológicas de la vegetación en cada ciudad son distintas (ver Tabla 2).

Resulta importante destacar que el modelo de vegetación de ENVI-Met incluye temperatura de hoja, evapotranspiración y sombra. Estas características interactúan con el modelo atmosférico, así como también con el modelo de suelo y de radiación. Las plantas en ENVI-Met 3.1 son modeladas como columnas de vegetación (ENVI-Met, 2017; HUTTNER, 2012; MOHAMAD; STEPHEN; MAHMOUD, 2010) y se caracterizan por diversos parámetros, en los que la LAD es uno de ellos. El perfil LAD cambia según la altura de los árboles. Los perfiles LAD proporcionados por ENVI-met son dibujados a mano y basados en sólo unos pocos perfiles de referencia. Aunque esto parece limitado, es una forma de medición muy viable (ENVI-MET, 2017).

En esta investigación, los árboles fueron extraídos de la base de datos; pero, además, se utilizó un enfoque analítico para comparar el perfil LAD seleccionado con el caso real. Según MEIR; GRACE; MIRANDA (2000), es posible ajustar el perfil LAD utilizando imágenes fotográficas. Por lo tanto, en este estudio, se evaluó una imagen digital hemisférica del cañón urbano de cada área y se calculó el FVC. Previamente, con el fin de validar la selección de árboles de la base de datos del simulador, se ajustó el valor FVC medido en el caso real, con las especies proporcionadas en la librería del ENVI-Met 3.1.

El modelo de simulación se ajusta con la radiación del día típico seleccionada en cada ciudad. Las simulaciones comenzaron a las 21:00 hora local. Fueron simulados cinco días (5 ciclos) y la estabilidad del modelo fue alcanzada en el cuarto día. Para verificar la exactitud del modelo teórico de ENVI-Met se comparó en ambas ciudades la curva de temperatura de aire simulada (S) con la observada $(\mathrm{O})$ en el punto 1 . Con estos datos fue calculado el error cuadrático medio (RMSE) y sus componentes sistemáticos (RMSES) y el no sistemático (RMSE), la desviación de las medias (MBE), y el Coeficiente de Determinación $\left(\mathrm{R}^{2}\right)$. Los resultados muestran una coincidencia similar entre los registros observados y simulados. El $\mathrm{R}^{2}$ cerca de 1 indica un buen ajuste de los modelos. Las magnitudes bajas del indicador RMSE y sus componentes RMSES y RMSEU sugieren que los escenarios simulados reflejan un buen ajuste de las condiciones microclimáticas observadas en las áreas de estudio (Figura 2).

Los datos de salida del programa ENVI-Met fueron calibrados por el receptor 2 (localizado en el mismo lugar del punto observado, Punto 2) y analizados por el receptor 1 (Punto 1) el cual fue localizado en el centro del cañón (Figura 1).

\section{Escenarios propuestos}

A partir del modelo teórico validado, fueron modelados diferentes escenarios en alta densidad por ser la condición más demandante en términos de reducir las temperaturas de aire exterior. Los escenarios propuestos poseen una relación de aspecto de ratio $\mathrm{H} / \mathrm{W}=1,5$. Esta relación representa en el área de estudio de la ciudad de Mendoza una altura edilicia de 27 metros ( 9 pisos), considerando un ancho de canal vial de 18 metros. Mientras que en el área de Campinas, con un ancho de canal vial de 30 metros, la altura edilicia corresponde a 45 metros de altura edilicia (15 niveles).

Tabla 2- Características de la vegetación utilizada en el modelo teórico en cada área

\begin{tabular}{c|c|c|c}
\hline Área & Característica & Altura & Índice de área foliar (LAD) \\
\hline \multirow{2}{*}{ Mendoza } & Árbol liviano & $15,0 \mathrm{~m}$ & LAD1: 0,$04 ; \mathrm{LAD} 6: 0,150 ; \mathrm{LAD} 10: 0,00\left(\mathrm{~m}^{2} / \mathrm{m}^{3}\right)$ \\
& Árbol denso & $15,0 \mathrm{~m}$ & LAD1: 0,$15 ;$ LAD6: 2,$15 ; \mathrm{LAD} 10: 0,00\left(\mathrm{~m}^{2} / \mathrm{m}^{3}\right)$ \\
\hline \multirow{3}{*}{ Campinas } & Césped & hasta $0,6 \mathrm{~m}$ & LAD1: 0,$30 ; \mathrm{LAD} 6: 0,30 ; \mathrm{LAD} 10: 0,30\left(\mathrm{~m}^{2} / \mathrm{m}^{3}\right)$ \\
& Cobertura densa & hasta $0,6 \mathrm{~m}$ & LAD1: 2,$50 ;$ LAD6: 2,$50 ;$ LAD10:1,50 $\left(\mathrm{m}^{2} / \mathrm{m}^{3}\right)$ \\
& Árbol denso & $10,0 \mathrm{~m}$ & LAD1: 0,$075 ;$ LAD6: 1,$150 ;$ LAD10: $0,00\left(\mathrm{~m}^{2} / \mathrm{m}^{3}\right)$ \\
\hline
\end{tabular}


Figura 2 - Curva de Calibración de Temperatura de Aire (Punto 1)

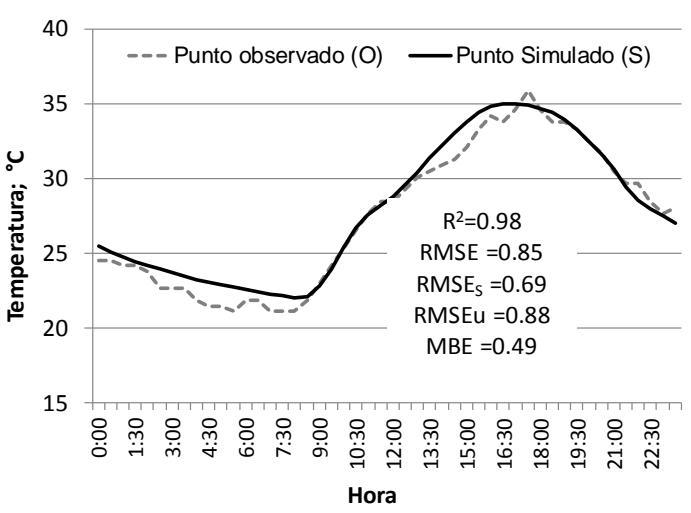

(a)

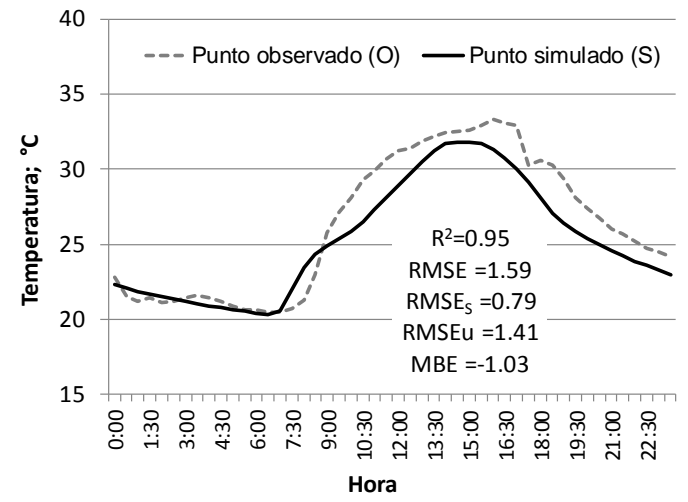

(b)
Fueron simuladas 9 configuraciones de escenarios que modifican sus rangos de albedo en materiales de la envolvente urbana - N1, N2, N3 - , porcentajes de vegetación $-0 \%, 20 \%$ y $60 \%$ - y sus respectivos FVC. Si bien la forma edilicia en este trabajo permanece constante $(\mathrm{H} / \mathrm{W}: 1,5)$ el porcentaje de vegetación es distinto en cada escenario, lo que impacta en la modificación del FVC (Figura 3). La Tabla 3 expresa las características de cada parámetro modificado y la codificación adoptada y en la Tabla 4 se detallan los valores de FVC de acuerdo a su porcentaje de vegetación para cada escenario evaluado.

\section{Análisis multivariado por componentes principales $(\mathrm{ACP})$ : descripción del método}

Con el objetivo de analizar el conjunto de datos simulados y las posibles asociaciones entre las variables se hizo un análisis de componentes principales. El Análisis de Componente Principal (ACP) es un análisis estadístico multivariado y como herramienta exploratoria permite revelar la existencia o no de relaciones entre las variables medidas y de las relaciones o agrupaciones entre muestras.

El ACP posibilita la investigación de un conjunto de variables correlacionadas y transforma el conjunto de variables originales en un nuevo conjunto de variables no correlacionadas denominadas como componentes principales, los cuales tienen propiedades especiales en términos de varianzas. Los componentes principales son combinaciones lineales de las variables originales y derivadas en orden decreciente de importancia. Así, el primer componente principal es la combinación lineal normalizada con varianza máxima y el segundo componente principal es la combinación lineal con variación máxima en dirección ortogonal al primer componente principal (RENCHER, 2002).

El ACP puede ser conducido a través de la matriz de correlación $(\mathrm{R})$ o por la matriz de covariancia (S). Para esta investigación se utilizó la matriz de correlación, la cual trabaja variables mensuradas en diferentes unidades (WILKS, 2006). En este estudio, los datos iniciales utilizados para el ACP provienen de las variables de los 9 escenarios de estudio para cada ciudad (Mendoza y Campinas) totalizando 18 escenarios. Para la realización de la ACP se reunieron en una matriz los datos de temperatura del aire cada 30 minutos de un día de medición de todos los escenarios de estudios y la tabulación de las variables: factor de visión del cielo (FVC), albedo de las paredes (ALB_Par), albedo de las cubiertas (ALB_Cob), albedo de pavimentos peatonales (ALB_Pav_Peat), albedo de pavimentos vehiculares (ALB_Pav_Veh), porcentaje de vegetación (VEG).

Los análisis multivariados contemplaron dos etapas: un análisis conjunto de las dos ciudades y un análisis individualizado de cada ciudad de estudio. Las mejores correlaciones son aquellas que muestran un mayor porcentaje de explicación de un fenómeno con menor número de factores discutidos.

\section{Resultados y análisis \\ Temperaturas de aire según escenario}

La Tabla 5 describe la temperatura de aire $\left({ }^{\circ} \mathrm{C}\right) \mathrm{de}$ acuerdo al escenario, para el punto 2 en cada ciudad. 
Figura 3 - Axonometrías de escenario de alta densidad en la ciudad de Mendoza y Campinas

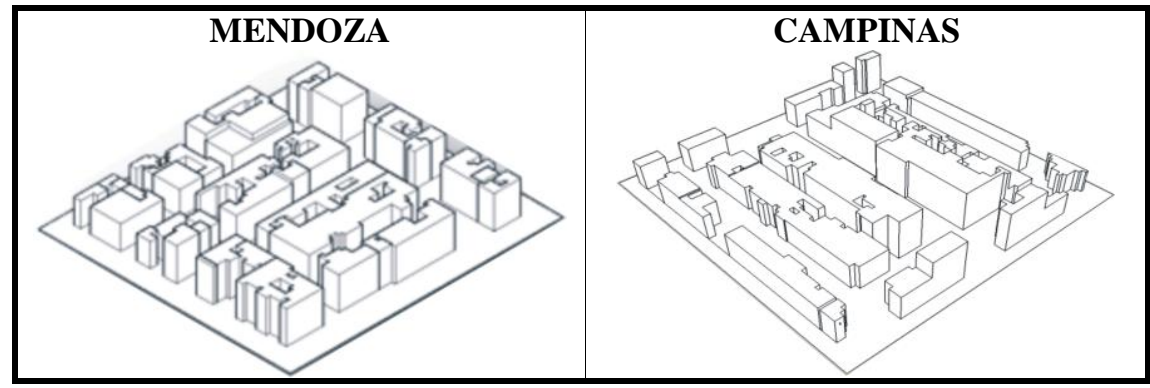

Tabla 3 - Características de los componentes modificados en cada escenario y codificación adoptada

\begin{tabular}{|c|c|c|c|c|c|}
\hline \multirow{2}{*}{\multicolumn{3}{|c|}{ PARÁMETROS }} & \multicolumn{3}{|c|}{ Vegetación urbana } \\
\hline & & & $0 \%$ & $20 \%$ *** & $60 \%{ }^{* * * *}$ \\
\hline \multirow{3}{*}{ 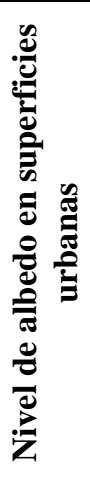 } & $\mathbf{N} 1^{*}$ & $\begin{array}{l}\text { Albedo cobertura de techo }(\text { ALB_Cob) }=0,30 ; \\
\text { Albedo de paredes (ALB_Par) }=0,20 ; \\
\text { Albedo pavimentos vehiculares }(\text { ALB_Via) }=0,2-0,30 ; \\
\text { Albedo pavimentos peatonales (ALB_Piso) }=0,2-0,40 \text {. }\end{array}$ & E1.a & E1.b & E1.c \\
\hline & $\mathbf{N 2}$ & $\begin{array}{l}\text { Albedo cobertura de techo }\left(\mathrm{ALB} \_\mathrm{Cob}\right)=0,80 ; \\
\text { Albedo de paredes }(\mathrm{ALB} \text { _Par) }=0,80 ; \\
\text { Albedo pavimentos vehiculares }(\mathrm{ALB} \text { _Via })=0,7 ; \\
\text { Albedo pavimentos peatonales }\left(\mathrm{ALB} \_\mathrm{Piso}\right)=0,7\end{array}$ & E2.a & E2.b & E2.c \\
\hline & N3 & $\begin{array}{l}\text { Albedo cobertura de techo (ALB_Cob) }=0,80 ; \\
\text { Albedo de paredes (ALB_Par) }=0,20 ; \\
\text { Albedo pavimentos vehiculares }(\text { ALB_Via) }=0,7 ; \\
\text { Albedo pavimentos peatonales }(\text { ALB_Piso })=0,7\end{array}$ & E3.a & E3.b & E3.c \\
\hline
\end{tabular}

Nota: $\left({ }^{*}\right)$ Corresponde a situación actual de nivel de albedo del área de estudio de ambas ciudades;

$\left.{ }^{* *}\right)$ Corresponde a situación actual de vegetación del área de estudio de la ciudad de Campinas; y

$\left.{ }^{* * *}\right)$ Corresponde a situación actual de vegetación del área de estudio de la ciudad de Mendoza.

Tabla 4 - Factor de visión de cielo (FVC) de los escenarios simulados para la ciudad de Mendoza y de Campinas

\begin{tabular}{lccc}
\hline \multirow{2}{*}{ ESCENARIOS } & \multicolumn{3}{c}{ FVC } \\
\cline { 2 - 4 } & $\mathbf{6 .}$ a & E. b & E. c \\
& 0,21 & $\mathbf{2 0} \%$ veg & 0 \% veg \\
\hline Mendoza & 0,33 & 0,37 & 0,38 \\
Campinas & 0,35 & 0,40 \\
\hline
\end{tabular}

Tabla 5 - Temperatura de aire $\left({ }^{\circ} \mathrm{C}\right)$ mínima (MIN), máxima (MAX), amplitud (AMPL) y promedio (PROM) según escenarios en Mendoza y Campinas

\begin{tabular}{c|l|l|l|l|l|l|l|l|l}
\hline TEMP AIRE $\left({ }^{\circ} \mathbf{C}\right)$ & \multicolumn{10}{c}{ MENDOZA } \\
\cline { 2 - 10 } & E3.a & E3.b & E3.c & E4.a & E4.b & E4.c & E6.a & E6.b & E6.c \\
\hline MIN & 23,1 & 23,7 & 23,9 & 23,5 & 23,9 & 24,3 & 22,9 & 23,3 & 23,7 \\
MAX & 33,7 & 34,9 & 35,3 & 34,7 & 35,4 & 35,5 & 32,1 & 33,5 & 33,8 \\
AMPL & 10,7 & 11,2 & 11,4 & 11,3 & 11,5 & 11,2 & 9,2 & 10,2 & 10,1 \\
PROM & 27,7 & 28,6 & 28,8 & 28,5 & 29,0 & 29,3 & 26,8 & 27,6 & 28,1 \\
\hline \multicolumn{10}{|c}{ CAMPINAS } \\
\hline MIN & 19,7 & 20,6 & 21,3 & 20,1 & 21,0 & 21,7 & 19,6 & 20,4 & 21,0 \\
\cline { 2 - 10 } & 27,6 & 30,6 & 32,5 & 29,2 & 31,5 & 32,6 & 25,7 & 28,5 & 30,4 \\
AMPL & 7,8 & 10,0 & 11,3 & 9,1 & 10,4 & 11,0 & 6,1 & 8,1 & 9,3 \\
PROM & 22,9 & 24,5 & 25,6 & 23,9 & 25,2 & 26,0 & 22,1 & 23,6 & 24,6 \\
\hline
\end{tabular}




\section{Análisis descriptivo}

Los resultados de las simulaciones de los 9 escenarios analizados para cada ciudad (total de 18 escenarios) constituyen el universo del análisis estadístico.

La Figura 4 grafica los valores de temperatura del aire máxima (Temp_Max), mínima (Temp_Min), amplitud térmica (Ampl_Térmica), Temperatura de aire media (Temp_Media) del total de escenarios simulados correspondientes a los puntos 2 señalados en la Tabla 2. La Figura 5 muestra las temperaturas del aire en los 18 escenarios simulados. Al comparar el total de los escenarios se verifica que la ciudad de Mendoza es más cálida que Campinas, a pesar de que en la situación actual de Mendoza el porcentaje de vegetación es mayor $(60 \%)$ que en Campinas $(20 \%)$. Las temperaturas de aire del total de los escenarios de Mendoza oscilan entre $24,0^{\circ}$ y $33,0^{\circ} \mathrm{C}$, mientras que en Campinas oscilan entre 20 y $29^{\circ} \mathrm{C}$.

Al evaluar la vegetación, bajo los mismos parámetros de albedo, se observa que los escenarios que tienen $60 \%$ de vegetación (E1.a, E2.a y E3.a) registran las menores temperaturas de aire urbano. En la ciudad de Mendoza incrementar la vegetación disminuye la temperatura de aire hasta $2,0^{\circ} \mathrm{C}$ y en Campinas hasta $5,0^{\circ} \mathrm{C}$ (Ver en Tabla 5: Temp_Max en E.3.a vs. E.3.c en Mendoza; E.1 a vs. E.1.c en Campinas).

Figura 4 - Diagramas de temperaturas máximas $\left({ }^{\circ} \mathrm{C}\right)$, temperaturas mínimas $\left({ }^{\circ} \mathrm{C}\right)$, temperatura media $\left({ }^{\circ} \mathrm{C}\right)$ y amplitud térmica $\left({ }^{\circ} \mathrm{C}\right)$ de Mendoza y Campinas

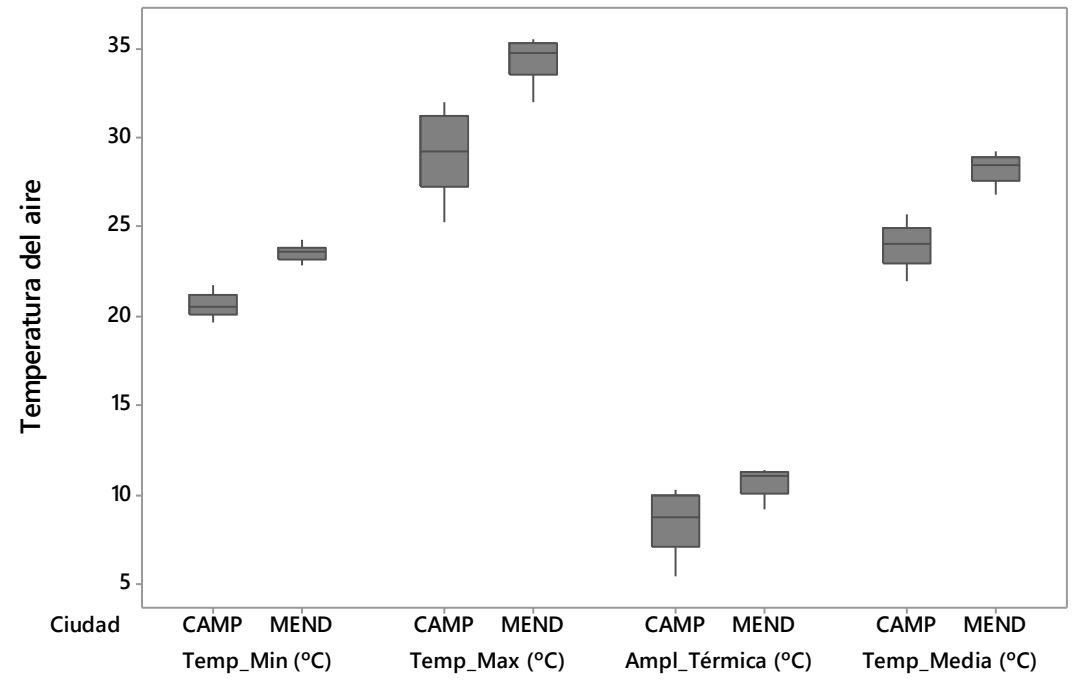

Figura 5 - Boxplot de temperatura del aire de escenarios de estudio de Mendoza y Campinas

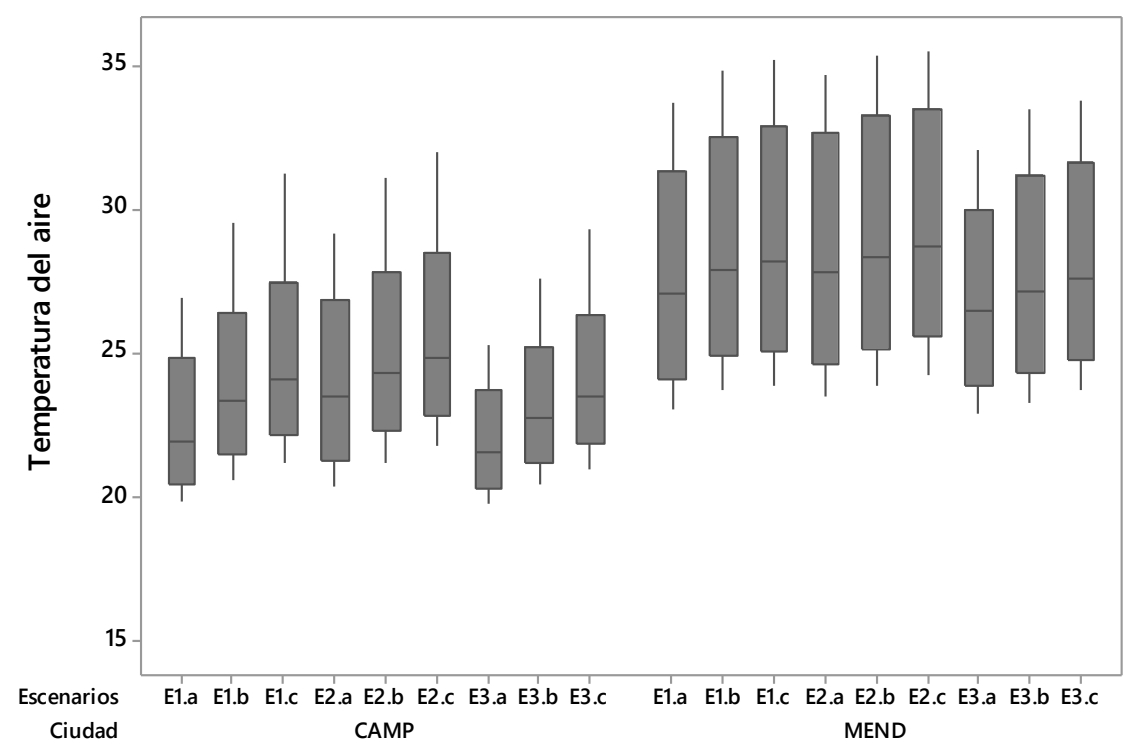

206 Alchapar, N. L.; Pezzuto, C. C.; Correa, E. N. 
Al evaluar los niveles de albedo de los materiales de las superficies urbanas, bajo los mismos porcentajes de vegetación, se observa que aumentar el albedo en todas las superficies de la envolvente (N2) eleva las temperaturas máximas del aire respecto a los escenarios con nivel de albedo N1 (E1.a, b, c versus, E2.a, b, c). En ambas ciudades se registran incrementos de hasta $1,0^{\circ} \mathrm{C}$. Mientras que los escenarios con rango de albedo $\mathrm{N} 3$, es decir alto albedo en superficies horizontales y bajo albedo en superficies verticales, registran menor temperatura de aire para todas las configuraciones ensayadas en ambas ciudades. (Ver en Tabla 5: E3.a, E3.b y E3.c). Se observan disminuciones de temperatura de aire de hasta 2,0 ${ }^{\circ} \mathrm{C}$ en Mendoza y de 3,0 ${ }^{\circ} \mathrm{C}$ en Campinas.

Es decir que en alta densidad la estrategia de incrementar el albedo en toda la envolvente (N2) resulta perjudicial durante el período de calentamiento, debido a que se disminuye la porción de visión efectiva al cielo dado por la altura edilicia quedando atrapada la radiación reflejada por las superficies con alto reflectancia solar. Estas múltiples reflexiones dentro del cañón vial aumentan la temperatura de las superficies y del aire circundante como consecuencia del incremento de la temperatura media radiante. Sin embargo, los escenarios con albedo alto en superficies horizontales y bajos en superficies verticales $(\mathrm{N} 3)$, registran menor temperatura de aire para todas las configuraciones ensayadas en ambas ciudades.

\section{Análisis multivariado de componentes principales}

La primera etapa del análisis contempló el conjunto de las variables: factor de visión del cielo (FVC), albedo de las paredes (ALB_Par), albedo de las cubiertas (ALB_Cob), albedo de los pavimentos vehiculares (ALB_Pav_Veh), albedo de pavimentos peatonales (ALB_Pav_Peat), porcentaje de vegetación (VEG) y las respectivas variaciones de temperatura del aire (TEMP) cada 30 minutos en un día de simulación.

La segunda fase del análisis contempló la adopción de un subconjunto de variables. Para ello, las variables referenciadas por los albedos (ALB_Par, ALB_Cob, ALB_Pav_Veh y ALB_Pav_Peat), se redujeron a un conjunto de variables únicas. Así se adoptó una nueva variable el Albedo Medio (ALB_Med), representada por las medias de las cuatro variables adoptadas.Según Jolliffe (1972, 1973) en análisis multivariados el empleo de un subconjunto, a partir de un gran número de características, no promueve cambios significativos en los resultados. Es ciertamente útil reducir el número de variables, si es posible, para las variables presentes que complican los datos, y no presentan ninguna información adicional. En consecuencia, la evaluación de un menor número de variables posibilita el ahorro de tiempo, tanto en la toma de datos y en los análisis computacionales, además de reducir los costos en análisis futuros. Muchos métodos son posibles para decidir qué variables se descartarán, pero en la práctica, la experiencia e intuición, a menudo desempeñan un papel en la selección.

Los gráficos siguientes ilustran las representaciones vectoriales de cada variable observada. Los vectores pueden ser observados según su magnitud y su posición relativa. A partir de los análisis de componentes principales las variables que tienen su representación vectorial con pequeña magnitud son poco explicadas. Las variables que están fuertemente y positivamente asociadas presentan sus vectores con dirección y sentido similares. Así el aumento de la magnitud de una variable ocasiona el aumento de la magnitud de la otra. En cambio, vectores con sentidos opuestos denota asociaciones fuertes negativas y los vectores que forman ángulos de $90,0^{\circ}$ no están correlacionados (SALGADO, 2006).

Como criterio se consideró que solamente los componentes principales que presentan un autovalor (varianza) superior a uno. Este criterio tiende a incluir pocos componentes cuando el número de variables es inferior a veinte, así que los componentes utilizados son los que sintetizan una varianza acumulada en torno al 70,0\% (NORUSIS, $1990^{2}$ apud ANDRADE; SILVEIRA; AZEVEDO, 2003, MARDIA, $1979^{3}$ apud VINICI, 2005).

\section{Evaluación conjunta de ambas ciudades}

En la Tabla 6 se observa que el modelo con dos componentes principales fue adecuado porque se explica el $71,5 \%$ de la variancia total. En el primer componente, PC1 $(1,789)$ y segundo componente, PC2 (1,702). Los componentes 3 y 4, PC3 $(0,9488)$ y PC4 $(0,1920)$, presentaron una varianza menor que uno, por lo que presentan una baja explicación.

Al verificar la contribución de las variables en la formación de los componentes (Tabla 7) se debe

\footnotetext{
${ }^{2}$ NORUSIS, M. S. SPSS Base System User's Guide. Chicago: SPSS, 1990

${ }^{3}$ MARDIA, K. V.; KENT, J. T.; BIBBY, J. M. Multivariate Analysis. London: Academic Press, 1979.
} 
observar que los valores en destaque son los que presentan una significancia cercana a 0,7 .

Es decir que, si tomamos como variable dependiente a la temperatura del aire, en el primer componente (PC1) las variables de mayor significancia fueron a la vegetación y el FVC (Figura 6). De ahí, el FVC está inversamente relacionado con la vegetación. Por lo tanto, a medida que se aumenta la proporción de vegetación disminuye el FVC y la temperatura. El segundo factor (PC2) está representado por el albedo de los materiales que componen el entorno urbano del canal evaluado. La temperatura del aire está directamente relacionada con el albedo medio de las superficies, es por ello que, incrementar el albedo medio aumenta la temperatura del aire.

Al evaluar los dos componentes, se verifica que las variables con alto peso en el componente 1 presentan una estrecha relación con los parámetros morfológicos, por lo tanto, pudiendo ser aquí denominado como componente morfológico. En la componente 2 , la variable albedo medio presenta los mayores valores, pudiendo ser nombrado como componente de los materiales urbanos (o componente tecnológico). Los resultados indican que en la estimación del microclima del cañón urbano cada variable presenta un peso diferente y deben ser evaluadas en conjunto.

\section{Evaluación individualizada por ciudad: Mendoza y Campinas}

Para profundizar los resultados anteriores, los conjuntos de muestras se analizaron por separado: simulaciones correspondientes a los escenarios con el subconjunto de albedo medio. (Tablas 8 y 9 y Figura 7).

Tabla 6- Resultados de los auto-valores, varianzas y varianzas acumuladas de las variables por Componentes Principales, basadas en los escenarios de estudio de ambas ciudades

\begin{tabular}{c|c|c|c|c}
\hline \multicolumn{5}{c}{ Matriz de correlación (TEMP, VEG, ALB_Med, FVC) } \\
\hline Estadísticos & PC1 & PC2 & PC3 & PC4 \\
\hline Auto-valor (Varianza) & $\mathbf{1 , 7 8 9 0}$ & $\mathbf{1 , 0 7 0 2}$ & 0,9488 & 0,1920 \\
Porcentaje Varianza (\%) & 44,7 & 26,8 & 23,7 & 4,8 \\
Varianza Acumulada (\%) & 44,7 & 71,5 & 95,2 & 100,0 \\
\hline
\end{tabular}

Tabla 7 - Valores de las variables de estudio en relación al primer y segundo Componente Principal, para ambas ciudades

\begin{tabular}{l|c|c}
\hline \multicolumn{1}{c|}{ Variables } & PC1 & PC2 \\
\hline TEMP & 0,101 & $\mathbf{- 0 , 7 4 4}$ \\
VEG & $\mathbf{- 0 , 7 1 0}$ & 0,035 \\
ALB_Med & 0,005 & $\mathbf{- 0 , 6 5 0}$ \\
FVC & $\mathbf{0 , 6 9 7}$ & 0,149 \\
\hline
\end{tabular}

Figura 6 - Componentes principales para las variables: temperatura (TEMP), porcentaje de vegetación (VEG), albedo medio (ALB_,Med) y factor de visión de cielo (FVC)

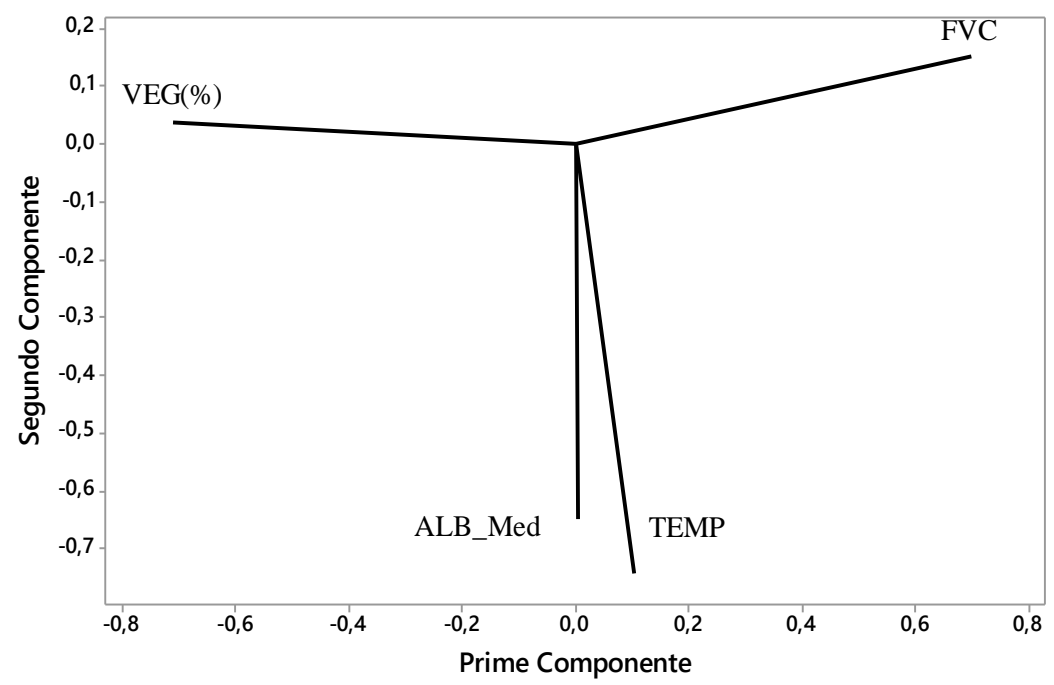

208 Alchapar, N. L.; Pezzuto, C. C.; Correa, E. N. 
Tabla 8 - Resultados de auto-valores, varianzas y varianzas acumuladas de las variables por Componentes Principales, basadas en los escenarios de estudio en la ciudad de Mendoza y de Campinas

\begin{tabular}{l|l|l|l|l|l}
\hline \multicolumn{5}{c}{ MENDOZA } \\
\hline Matriz de correlación (TEMP, VEG, MEAN_ALB, FVC) & \multicolumn{1}{c}{ PC1 } & \multicolumn{1}{c}{ PC2 } & \multicolumn{1}{c}{ PC3 } & \multicolumn{1}{c}{ PC4 } \\
\hline Auto-valor (Varianza) & 1,9774 & 1,0219 & 0,9504 & 0,0502 \\
Porcentaje Varianza (\%) & 0,494 & 0,255 & 0,238 & 0,013 \\
Varianza Acumulada (\%) & 0,494 & $\mathbf{0 , 7 5 0}$ & 0,987 & 1 \\
\hline \multicolumn{7}{c|}{ CAMPINAS } & \multicolumn{1}{c}{ PC2 } & \multicolumn{1}{c}{ PC3 } & \multicolumn{1}{c}{ PC4 } \\
\hline Matriz de correlación (TEMP, VEG, ALB_Medio, FVC) & PC1 & 1,9899 & 1,0491 & 0,8071 & 0,1539 \\
\hline Auto-valor (Varianza) & 0,497 & 0,262 & 0,202 & 0,038 \\
Porcentaje Varianza (\%) & 0,497 & $\mathbf{0 , 7 6 0}$ & 0,962 & 1 \\
Varianza Acumulada (\%)
\end{tabular}

Tabla 9 - Valores de las variables de estudio en relación al primer y segundo Componente Principal, para la Ciudad de Mendoza y Campinas

\begin{tabular}{l|c|c|c|c}
\hline \multirow{2}{*}{ Variables } & \multicolumn{2}{|c|}{ PC1 } & \multicolumn{2}{c}{ PC2 } \\
\cline { 2 - 5 } & MEND & CAMP & MEND & CAMP \\
\hline TEMP & 0,166 & 0,358 & 0,546 & $-0,414$ \\
VEG & $\mathbf{- 0 , 6 9 7}$ & $\mathbf{- 0 , 6 6 3}$ & 0,067 & $-0,131$ \\
MEAN_ALB & 0,006 & 0,038 & $\mathbf{0 , 8 3 2}$ & $\mathbf{- 0 . 8 8 9}$ \\
FVC & $\mathbf{0 , 6 9 7}$ & $\mathbf{0 , 6 5 6}$ & $-0,070$ & 0,146 \\
\hline
\end{tabular}

Figura 7 - Componentes principales para las variables: temperatura (TEMP), porcentaje de vegetación (VEG), albedo medio (MEAN_ALB) y factor de visión de cielo (FVC) en el área de estudio de la ciudad de Mendoza y de Campinas

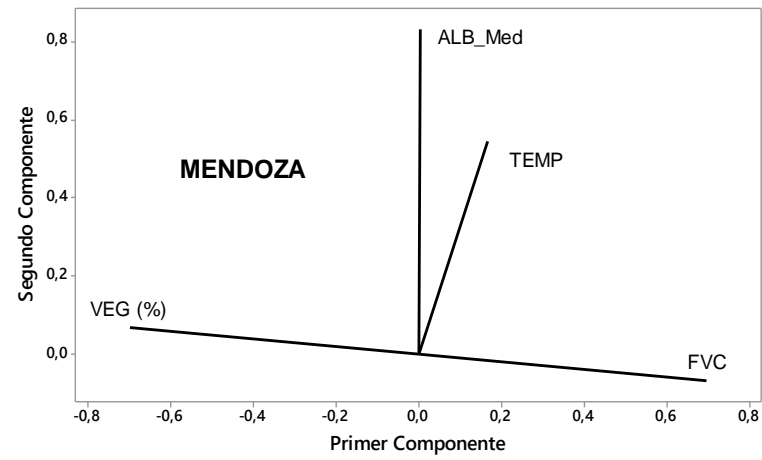

Se observa un comportamiento análogo. Es decir, en la situación de verano, el $75,0 \%$ y $76,0 \%$ del comportamiento de la temperatura del aire en los cañones urbanos de la ciudad de Mendoza y de Campinas, respectivamente, puede ser explicado por dos factores principales. Es decir, las varianzas superiores al valor 1, son representadas por el primer componente - PC1 $(1,9774)$ y segundo componente - PC2 $(1,0219)$ para Mendoza y PC1 $(1,9899)$ y PC2 $(1,0491)$ para Campinas. Los componentes 3 y 4, PC3 y PC4, en ambas ciudades tienen variación menor, por lo tanto, presentan bajo grado de explicación.

En ambas ciudades, el primer componente está relacionado con el porcentaje de vegetación y el factor de visión del cielo, los cuales presentaron significancia cercana a 0,7 . Estas dos variables

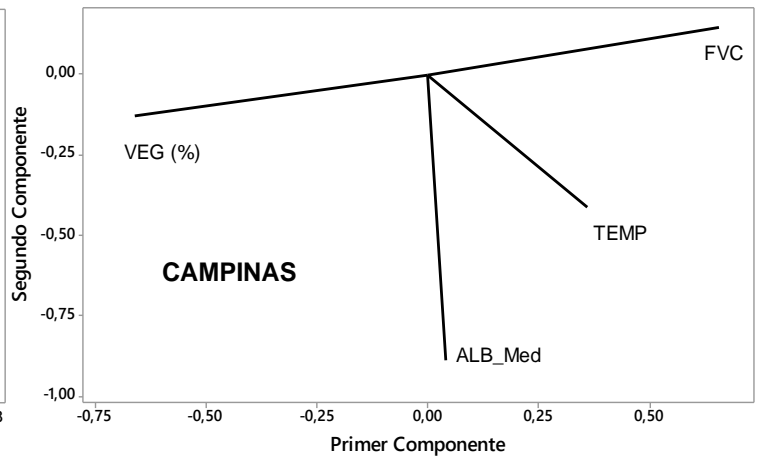

denotan asociaciones fuertes negativas, o sea mayor magnitud de porcentaje de vegetación ocasiona menor magnitud de factor de visión del cielo y menor temperatura, y viceversa

El segundo componente está relacionado con el albedo medio de las superficies urbanas. Resultando para la configuración urbana analizada (mayor altura edilicia) una relación directamente proporcional. Esto implica que cuando el albedo combinado se incrementa la temperatura de los canales viales se incrementa.

En la evaluación de los dos componentes nuevamente se verifica que las variables con un alto peso en el componente 1 están representadas por los parámetros morfológicos. Sin embargo, en el componente 2 solamente el albedo medio presentó mayor significancia pudiendo, en este 
caso, nombrar como componentes de los materiales urbanos o componente morfológico.

\section{Discusión}

Cabe destacar que en la bibliografía internacional existen numerosas investigaciones que afirman que elevar el albedo medio de las ciudades disminuye las temperaturas urbanas. Por ejemplo, Akbari, Matthews y Seto (2012) afirman que elevar el albedo de las superficies urbanas en aproximadamente 0,1 , en promedio, puede reducir la temperatura urbana de verano en $1,0{ }^{\circ} \mathrm{C}$ y mejorar calidad del aire en las 100 ciudades más pobladas del mundo.

Sin embargo, este trabajo muestra que el paradigma de aumentar el albedo en todas las áreas de la ciudad sin distinguir densidad, orientación, ni posición relativa del material (piso, techo y fachada) puede causar serios problemas de sobrecalentamiento dentro del cañón urbano. En coincidencia con hallazgos de Yang, Wang y Kaloush (2015), quienes demostraron que la utilización de materiales con alto albedo sobre las envolventes es un método eficaz para reducir la temperatura y el consumo de energía en las ciudades, pero el impacto del uso indiscriminado sobre la sostenibilidad ambiental está insuficientemente explorado. Erell et al. (2014) reportó que el uso extendido de alto albedo en superficies urbanas, en ciudades de clima cálido, pueden disminuir las temperaturas de aire, pero incrementan las cargas de calor radiativo. Como resultado se compromete el confort de los peatones.

En este sentido, los análisis estadísticos realizados en la presente investigación, demuestran que elevar el albedo medio del canal vial de alta densidad incrementa la temperatura de aire en situación de verano. En contraste con análisis del comportamiento térmico de canales de baja densidad (H/W: 0,3), en donde Alchapar, Pezzuto y Correa (2017) evidenciaron reducciones de temperatura de aire entre 1,0 y $1,5{ }^{\circ} \mathrm{C}$. Es decir, que la aplicación extendida de incrementar el albedo en recientes de alta densidad debe ser analizado de forma particular, según morfología y contexto climático.

Cabe destacar que en esta investigación se ha considerado que el valor de albedo medio es el resultado del promedio ponderado por unidad de superficie, de todos los valores de las superficies de la envolvente del espacio urbano analizado (piso, techo y fachada). En donde las superficies verticales - fachadas - tienen mayor peso que las horizontales en relación con la superficie total, por lo que el albedo de las fachadas o las superficies verticales en los escenarios con $\mathrm{H} / \mathrm{W}$ : 1,50 (situaciones de alta densidad edilicia) tienen mayor peso sobre el valor del albedo medio.

En esa línea de ideas, investigaciones previas llevadas adelante por Alchapar, Pezzuto y Correa (2017) demostraron que incrementar el albedo en materiales de fachadas eleva las temperaturas de aire y media radiante dentro de un cañón urbano de alta densidad, debido a que las múltiples reflexiones quedan atrapadas en cañones urbanos profundos. Por ejemplo, en la ciudad de Mendoza en un canal vial de 18 metros de ancho, a partir de los 12 metros de altura de fachada (H/W: 0,66), es necesario mantener niveles de albedo inferiores a 0,50 para conseguir disminuir las temperaturas de un cañón urbano. Cada 0.1 de incremento de albedo en fachadas la temperatura de aire se eleva $0,5^{\circ} \mathrm{C}$ en situación de verano (ALCHAPAR; CORREA, 2016).

Brender y Lindsey (2006) evaluaron las interacciones térmicas entre materiales y superficies reflectantes con objetos cercanos. La interacción no tiene un gran efecto en áreas abiertas, pero tiende a ser más severa en centros urbanos muy densos donde las paredes están cerca de las calles y tienen así un mayor poder de calentamiento debido a la luz solar reflejada desde las superficies adyacentes. Qin (2015) recomienda que los pavimentos reflectantes solo se usen si un cañón urbano tiene una relación de aspecto no mayor a 1.0. Sin embargo, en el esquema urbano abierto (ancho de calles superiores a $18 \mathrm{~m}$ ) y clima árido como, por ejemplo, la ciudad de Mendoza, Achapar y Correa (2016) afirman que el uso de pavimentos reflectantes es favorable hasta una relación de aspecto $\mathrm{H} / \mathrm{W}=1.5 \mathrm{si}$ el albedo de las paredes es bajo (albedo ponderado $=0,65$ ). Los cañones urbanos vegetados con altos niveles de albedo en techos y pavimentos y bajos niveles en fachada resultan los más eficientes.

Lo planteado hasta el momento sugiere nuevas preguntas de investigación. El nivel de albedo óptimo en un canal vial se consigue alcanzando una reflectividad solar media de toda la envolvente urbana, o resulta de la distribución de albedo relativa a la posición de cada material presente en un recinto urbano (piso, fachada, techo).

\section{Conclusiones}

A partir de la evaluación individualizada de los escenarios de ambas ciudades, se verificó que las variables controlables -porcentajes de vegetación y nivel de albedo- tienen un impacto directo sobre las temperaturas de aire de los cañones urbanos. No obstante, si se mantienen los mismos parámetros morfo-materiales, las variables 
incontrolables - radiación solar, humedad, velocidad del viento, nubosidad, etc. - determinan el grado de intensidad del efecto de cada estrategia en las dos ciudades estudiadas.

Este estudio permitió verificar que los resultados obtenidos en la simulación son coherentes con lo que se indica en el Análisis Multivariado de Componentes Principales. El porcentaje de la vegetación y el albedo medio de las superficies opacas urbanas explica el 71,5 \% de los comportamientos dentro de los canales viales evaluados.

En base a los análisis estadísticos, observamos que el incremento de la vegetación en todos los escenarios ofrece las mejores posibilidades para mejorar las condiciones térmicas urbanas. Por lo tanto, el aumento en la vegetación de 0 a $60 \%$ podría disminuir la temperatura media en Mendoza hasta $2,0^{\circ} \mathrm{C}$, mientras que en Campinas hasta 5,0 ${ }^{\circ} \mathrm{C}$. El aumento en el porcentaje de vegetación ofrece beneficios para ambas ciudades, sin embargo, el efecto en Campinas es mayor que en Mendoza, puede deberse a la anatomía y fisiología de las especies de árbol en cada ciudad. En climas templados, como es el caso de Campinas, el follaje es denso en comparación con las áreas de clima árido, como Mendoza. Bajo las mismas condiciones de morfología urbana y disposición forestal, las diferencias en la magnitud de reducción de la temperatura podrían ser explicadas por las interacciones entre la anatomía y fisiología del árbol con otras variables: radiación solar, velocidad de viento, humedad absoluta, etc., de cada ciudad.

Al evaluar el nivel del albedo de las superficies opacas se observó que aumentar el nivel en todas las superficies (nivel $\mathrm{N} 2$ ) eleva hasta $1,0^{\circ} \mathrm{C}$ las temperaturas de aire de escenarios de alta densidad en Campinas y en Mendoza. No obstante, en el caso de los escenarios con alto albedo en superficies horizontales y bajo albedo en verticales (E3), se registraron las menores temperaturas urbanas. Reducciones de $2,0{ }^{\circ} \mathrm{C}$ en la ciudad de Mendoza y de 3,0 ${ }^{\circ} \mathrm{C}$ en Campinas.

Es decir que, en alta densidad, incrementar el albedo en superficies verticales es una decisión que debe ser analizada en cada situación particular. Por el contrario, elevar el albedo de los materiales de las superficies horizontales es una estrategia que siempre brinda beneficios térmicos en el área donde se aplica.

Los resultados muestran posibilidades concretas de reducción de los consumos de energía en ambas ciudades, así como promover una mejora de las condiciones térmicas urbanas. En próximos trabajos se calcularán los beneficios energéticos que conllevan las mencionadas reducciones de temperatura de aire urbana. Lo señalado confirma la importancia de contar con modelos matemáticos que analicen las posibles relaciones entre la temperatura del aire y parámetros morfológicos y materiales, como una herramienta de decisión para el desarrollo urbano ambientalmente sustentable.

\section{Referencias}

ABREU-HARBICH, L. V.; LABAKI, L. C.; MATZARAKIS, A. Thermal Bioclimate in Idealized Urban Street Canyons in Campinas, Brazil. Theoretical and Applied Climatology, v. 115, p. 333-340, 2014.

AKBARI, H.; MATTHEWS, H. D.; SETO, D. The Long-Term Effect of Increasing the Albedo of Urban Areas. Environmental Research Letters, v. 7, n. 2,2012.

AKBARI, H.; MENON, S.; ROSENFELD, A. Global Cooling: increasing world-wide urban albedos to offset CO2. Climatic Change, v.94, n. 3/4, p. 275-286, 2009.

ALCHAPAR, N. et al. Impact of Cooling Strategies on Urban Temperatures in Warm Climates Cities: the case of Campinas, Brazil and Mendoza, Argentina. Theoretical and Applied Climatology, v. 130, n. 1/2, p. 35-50, 2017.

ALCHAPAR, N.; CORREA, E. The Use of Reflective Materials as a Strategy For Urban Cooling in an Arid "OASIS" City. Sustainable Cities and Society, v. 27, p. 1-14, 2016.

ALCHAPAR, N.; PEZZUTO, C.; CORREA, E. Análise Multivariada Como Ferramenta Para Orientar o Desenho de Espaços Urbanos Termicamente Aptos: estudo de caso da cidade de Campinas, Brasil e de Mendoza, Argentina. In: ENCONTRO NACIONAL E LATINOAMERICANO DE CONFORTO NO AMBIENTE CONSTRUÍDO, Camburiú, 2017. Anais... Camburiú: ANTAC, 2017.

\section{ALI-TOUDERT, F. Dependence of Outdoor Thermal Comfort on Street Design in Hot and Dry Climate. Freiburg, 2005. 224 f. Dissertation - Meteorologischen Institutes der Universität Freiburg, Freiburg, 2005.}

ANDRADE, E. M. de, SILVEIRA, S. S., AZEVEDO, B. M. de. Investigação da Estrutura Multivariada da Evapotranspiração na Região Centro Sul do Ceará pela Análise de Componentes Principais. Revista Brasileira de Recursos Hídricos, v. 8, n.1, p. 39-44, 2003. 
ARNFIELD, A. An Approach to the Estimation of the Surface Radiative Properties and Radiation Budgets of Cities. Physical Geography, v. 3, n. 2, p. 97-122, 1982.

BRENDER, D.; LINDSEY, T. Effect of Rooftop Exposure in di rect Sunlight onConduit Ambient Temperatures. Industry Applications

Transactions, v. 44, n. 6, p. 1872-1878, 2006.

BRUSE, M.; FLEER, H. Simulating SurfacePlant-Air Interactions Inside Urban Environments With a Three Dimensional Numerical Model. Environmental Modelling \& Software, v.13, n. 4/4, p. 373-384, 1998.

CHEN, Y. et al. Estimating the Relationship Between Urban Forms and Energy Consumption: a case study in the Pearl River Delta, 2005-2008.

Landscape and Urban Planning, v. 102, n. 1, p. 33-42, 2011.

DACANAL, C. Fragmentos Florestais Urbanos e Interações Climáticas em Diferentes Escalas: estudos em Campinas, SP. Campinas, 2011. $220 \mathrm{f}$. Tese (Doutorado em Engenharia Civil) - Faculdade de Engenharia Civil, Arquitetura e Urbanismo, Universidade Estadual de Campinas, Campinas, 2011.

ENVI-MET. ENVI-met 3.1 Manual Contents. Disponível em: <http://www.envimet.info/documents/onlinehelpv3/helpindex.htm>. Acesso em: 3 jul.2017.

ERELL, E.et al. Effect of High-Albedo Materials on Pedestrian Heat Stress in Urban Canyons.

Urban Climate, v. 10, n. 2, p. 367-386, 2014.

GIVONI, B. Passive and Low Energy Cooling of Building. New York: John Wiley \& Sons, 1994.

HUTTNER, S. Further Development and Application of the 3D Microclimate Simulation ENVI-met. Mainz, 2012. 137 f. Dissertação (Mestrado) - Johannes Gutenberg-Universität, Mainz, 2012.

JOLLIFFE, I. Discarding Variables in a Principal Component Analysis; I artificial data. Journal of the Royal Statistical Society, v. 21, n. 2, p. 160173, 1972.

JOLLIFFE, I. Discarding Variables in a Principal Component Analysis: II real data. Journal of the Royal Statistical Society,v. 22, n. 1, p. 21-31, 1973.

KOLOKOTRONI, M.; GIRIDHARAN, K. Urban Heat Island Intensity in London: an investigation of the impact of physical characteristics on changes in outdoor air temperature during Summer. Solar Energy, v. 82, n. 11, p. 986-998, 2008.
MEIR, P.; GRACE, J.; MIRANDA, A. Photographic Method to Measure the Vertical Distribution of Leaf Area Density in Forests. Agricultural and Forest Meteorology, v. 102, n. 2/3, p. 105-111, 2000.

MINELLA, F. C. O.; HONJO, S.; KRÜGER, E. L. Estratégias de Melhoria do Ambiente Térmico Diurno em Situação de Verão de Uma Fração Urbana da Cidade de São Paulo. Ambiente Construído, Porto Alegre, v. 12, n. 4, p. 139-158, out./dez. 2012.

MINELLA, F. C. O.; KRÜGER, E. L. Proposição do Índice "Fração Vegetada" e Sua Relação Com Alterações na Temperatura do Ar e no Conforto Térmico no Período Diurno e em Situação de Verão Para Curitiba. Ambiente Construído, Porto Alegre, v. 17, n. 1, p. 353-371, jan./mar. 2017.

MIRZAEI, P.; HAGHIGHAT, F. Approaches to Study Urban Heat Island: abilities and limitations. Building and Environment, v. 45, n. 10, p. $2192-$ 2201, 2010.

MOHAMAD, F.; STEPHEN, S.; MAHMOUD, Y. LAI Based Trees Selection For Mid Latitude Urban Developments: a microclimatic study in Cairo, Egypt. Building and Environment.v.45, n. 2, p. 345-357, 2010.

MUNIZ, L. P.; PEZZUTO, C. C. O Efeito dos Parâmetros Urbanísticos na Variação

Microclimática de Um Cânion Urbano. In: ENCONTRO NACIONAL DE TECNOLOGIA DO AMBIENTE CONSTRUÍDO, 16., São Paulo, 2016. Anais... Porto Alegre: ANTAC, 2016.

OKE, T. The Energetic Basis of the Urban Heat Island. Quarterly Journal of the Royal Meteorological Society, v. 108, n. 445, p. 1-24, 1982.

PEZZUTO, C. C. Avaliação do Ambiente Térmico nos Espaços Urbanos Abertos: estudo de caso em Campinas, SP. Campinas, 2007. $182 \mathrm{f}$. Tese (Doutorado em Engenharia Civil) - Faculdade de Engenharia Civil, Arquitetura e Urbanismo, Campinas, Universidade Estadual de Campinas, Campinas, 2007.

QIN, Y. Urban Canyon Albedo and Its Implication on the Use of Reflective Cool Pavements. Energy Buildings, v. 96, p. 86-94, 2015.

RENCHER, A. Methods of Multivariate Analysis. $2^{\text {nd }}$. ed. Canada: John Wiley \& Sons, Interscience Publication, 2002.

RIZWAN, A.; DENNIS, Y.; LIU C. A Review on the Generation, Determination and Mitigation of Urban Heat Island. Journal of Environmental Sciences, v. 20, n. 1, p.120-128, 2008. 
SALGADO, D. A. Modelo Estatístico Para Predição de Bem-Estar de Reprodutoras de Frango de Corte Baseado em Dados de Ambiente e Análise do Comportamento. Campinas, 2006. Dissertação (Mestrado em Engenharia Agrícola) - Faculdade de Engenharia Agrícola, Universidade Estadual de Campinas, Campinas, 2006.

SANTAMOURIS, M. et al. Passive and Active Cooling For the Outdoor Built Environment: analysis and assessment of the cooling potential of mitigation technologies using performance data from 220 large scale projects. Solar Energy, v. 154, n. 15, p. 14-33, 2017.

SANTAMOURIS, M.; SYNNEFA, A.;

KARLESSI, T. Using Advanced Cool Materials in the Urban Built Environment to Mitigate Heat Islands and Improve Thermal Comfort Conditions. Solar Energy, v. 85, n. 12, p. 3085-3102, 2011.

SHASHUA-BAR, L.; TSIROS, I. X.; HOFFMAN M. E. A Modeling Study For Evaluating Passive Cooling Scenarios in Urban Streets With Trees: case study, Athens, Greece. Building and Environment, v. 45, n. 12, p. 2798-2807, 2010.
SHASHUA-BAR, L.; TSIROS, I.; HOFFMAN, M.; Passive cooling design options to ameliorate thermal comfort in urban streets Building and Environment, v. 57, p.110-119, 2012.

STEWART, I. D.; OKE, T. R. Local Climate Zones for Urban Temperature Studies. Bulletin of the American Meteorological Society, v. 93, n. 12, p. 1879-1900, 2012.

UNITED STATES ENVIRONMENTAL PROTECTION AGENCY. Heat Island Effect. Disponível em: <http://www.epa.gov/heatisland/>. Acesso em: 15 maio 2015.

VICINI, L. Análise Multivariada da Teoria à Prática. Santa Maria, 2005. Monografia (Especialização em Estatística e Modelagem Quantitativa) - Departamento de Estatística, Universidade Federal de Santa Maria, Santa Maria, 2005.

WILKS, D. S. Statistical Methods in the Atmospheric Sciences. $2^{\text {nd }}$. ed. California: Elsevier Academic Press Publication, 2006.

YANG, J.; WANG, Z.; KALOUSH, K.

Environmental Impacts of Reflective Materials: is high albedo a "silver bullet" for mitigating urban heat island? Renewable and Sustainable Energy Reviews, v. 47, p. 830-843, 2015.

Noelia Liliana Alchapar

CCT CONICET | Instituto de Ambiente, Hábitat y Energía | Av. Ruiz Leal, s/n, Parque General San Martín | Mendoza - Argentina | CP 5500 | Tel.: + 54 (261) 524310 | E-mail:nalchapar@mendoza-conicet.gob.ar

\section{Cláudia Cotrim Pezzuto}

Programa de Postgrado em Sistemas de Infraestructura Urbana | Pontifícia Universidade Católica de Campinas | Rodovia Dom Pedro I, km 136, Parque das Universidades | Campinas - SP - Brasil | CEP 13086-900 | Tel.: (19) 3343-7000 | E-mail: claupezzuto@gmail.com

\section{Erica Norma Correa}

CCT CONICET | Instituto de Ambiente, Hábitat y Energía | E-mail: ecorrea@mendoza-conicet.gob.ar

Revista Ambiente Construído

Associação Nacional de Tecnologia do Ambiente Construído

Av. Osvaldo Aranha, $99-3^{\circ}$ andar, Centro Porto Alegre - RS - Brasil CEP $90035-190$

Telefone: +55 (51) 3308-4084

Fax: +55 (51) 3308-4054

www.seer.ufrgs.br/ambienteconstruido

E-mail: ambienteconstruido@ufrgs.br 